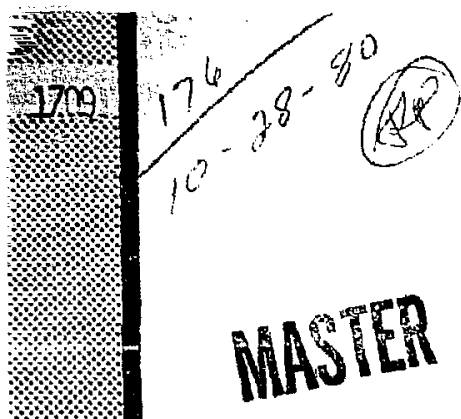

Dr. 1880

OCTOBER 1980

PPPL-1709

UC- $20 \mathrm{~g}$

\title{
ANOMALOUS TRANSPORT DUE TO SHEAR-ALFVEN WAVES
}

BY

W. W, LEE, M, S, CHANCE,

AND H. OKUDA

\section{PLASMA PHYSICS LABORATORY}

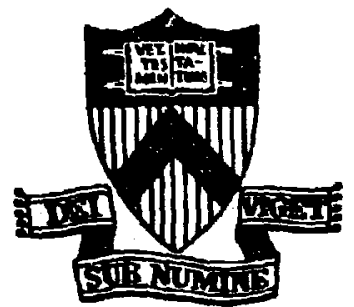

DISTAIBUTOOS OF THIS OOCUMEHT IS UMLIMTED

\section{PRINCETON UNIVERSITY PRINCETON, NEW JERSEY}

\footnotetext{
This mork was supported by the U.S. Department of Energy Contraet Ka. DE-ACO2-76-CHC 3073. Repraduceion, translation, publication, use and disposal, ir, whole or in part. by "or for the United Seates government is permitted.
} 
Anomalous Transport Die to Shear-AlEven Waves

by

W. W. Lee, M. S. Chance, and H. Okuda

Plasma Physics Laboratory, Princeton University

Princeton, New Jersey 08544

\begin{abstract}
The behavior of sheat-Alfven eigenmodes and the accompanied anomalous transport have been investigated. In the particle simulation, equilibrium thermal fluctuations associated with the eigennodes have been observed to nullify the zeroth-order shear near the rational surface through the inciced second-order eddy curxent, and, in turn, give rise to the formation of magnetic islands which cause rapid electron energy transport in the region. The theoretical vertfication of the observed behavior is discussed.
\end{abstract}

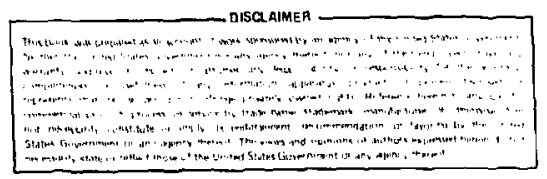


Since the recent recognition that magnetic field fluctuations associated with low-Erequency microinstabilities may be responsible for the anomalous electron energy transport in tokamaks, ${ }^{1-3}$ various linear stabj lity analyses have been performed $d^{3-5}$ to determine the onset conditions for the drift-Alfven waves, which are thought to be responsible for the experimental observation..$^{6-8}$ It has been shown that, in a sheared slab, drift-alfven eigenmodes which include the Einite- $\beta$ modified branch and the shear-alfven branch, are always stable for a collisionless plasma.4,5 This prediction prompted us to study the problem using a 2 1/2-dimensional finite- 3 particle simulation code. 9 The simulation results have indicated that the shear-Alfven eigenrodes indeed exist. The eigenmodes have the tearing-type parity of odd and even $A_{n}$, and are localized near the rational surface $\left(k_{\|}=0\right)$. Although the modes are linearly darped, finite-amplitude fluctuations associated with the radial eigenmodes with $k_{Y} p_{s}=1$ have been observed. They are the equilibrium thermal fluctuations representing the balance between the Cherenkov emission and the damping rate. Mode coupling between aifferent radial eigenmodes may also play a role here. These eluctuations, in turn, have produced a second-order eddy current which reduces shear near the ratinnal surface. A theoretical analysis, based on the quasilinear treatment of the second-order drift kinetic equation, has verified its existence. The saturation takes place when the zeroth-order current as well as the shear are completely nullified in the region. Consequently, the fluctuations in $A_{\|}$give rise to the formation of magnetic Islands with several $\rho_{i}$ 's in width, and 
rapid electron energy transport across the rational surface follows. In this letter, we will present the simulation results in detail together with their theoretical interpretations.

Let us first describe the linear formalism of drift-alfven waves relevant to our problem. For a low-B and collisionless plasma in a sheared slab where Eluctuations are proportional to exp(iky - iwt), the second order radial eigennode equations for the electrostatic potential $\phi$ and the vector potential $A_{\|}$, obtained from quasineutrality condition and Ampere's law, can be written as $4,5,10$

$$
\begin{aligned}
& \phi^{\prime \prime}=\left(\sigma / x_{A}^{2}\right)(\psi / x-\phi)+b_{s} \phi . \\
& \psi^{\prime}=(\sigma / x)(\psi / x-\phi)+b_{s} \psi .
\end{aligned}
$$

Here the independent variable $x$ is normalized by $\rho_{s}\left[=\left(\mathrm{T}_{e} / \mathrm{m}_{1}\right)^{1 / 2} / \omega_{c i}\right]$ $\psi=w_{A} / k_{i}^{\prime} c, k_{i}^{\prime}=k_{y} O_{s} / L_{s}$ where $L_{s}$ is the shear length, $b_{s}=k_{y}^{2} \rho_{s}^{2}, x_{A}=11$ $\left.+w^{*} / \tau w\right)^{1 / 2} w / x_{\|}^{\prime} v_{n}$ where $w^{\star}$ is the electron diamagnetic arift frequency, $v_{A}=$

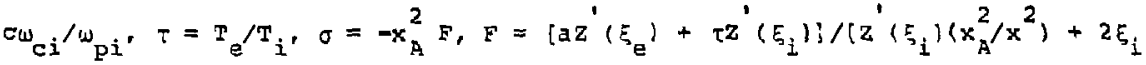
$\left.z\left(\xi_{j}\right)\right], a=\left(1-\omega^{*} / \omega\right) /\left(1+\omega^{*} / \tau \omega\right), \xi_{\alpha}=\omega / \sqrt{2} k_{n}^{\prime} x v_{t \alpha}$ where $\alpha$ denotes species, $v_{t \alpha}=\left(T_{\alpha} / m_{\alpha}\right)^{1 / 2}$ and $z$ is the usual plasma dispersion function. Equations (1) and (2) are valid in the IInst of $k^{2} p_{i}^{2}<i 1$ where $p_{i}=v_{t i} / w_{c i}$. Because of the behavior of $\psi / x$ - $\phi$ near the rational surface $(x=0)$, it $1 s$ evident these equations axe not anerable to numerical solutions. Since their WKB salutions indicate that both $\psi / x-\phi$ and $\phi-x \psi / x_{A}^{2}$ vanish for large $x$, 
Eqs. (1) and (2) can be rewritten, with a new variable $\$=\phi-x \psi / x_{A}^{2}$, as

$$
\begin{aligned}
& y^{\prime}-b_{S} \xi+\left(2 / x_{A}^{2}\right) \psi^{\prime}=0 \\
& \psi^{\prime \prime}+Q \psi+(\sigma / x) \phi=0
\end{aligned}
$$

where $Q=\sigma / x_{A}^{2}-\sigma / x^{2}-b_{s}$. These equations are easily solved by a standard Eourth-order shooting code. Since we are only interested in the spatially evanescent solutions, the wKB starting conditions at large $x$ are $(\phi, \phi, \psi, \psi '$ ') $=(0,0,1,-\sqrt{Q})$ and $\left(-x / x_{A}^{2}, \sqrt{b_{S}} / x_{A}^{2}, 1,-\sqrt{b_{s}}\right) \cdot$ The boundary conditions at $x=0$ are $\$=0, \psi \prime=0$. Furthermore, Egs. (3) and (4) can de decoupled through a perturbative scheme and, to the lowest order, the eigenfrequencies of the system can be determined by $y^{\prime}+Q y=0,11$ where $Q$ is defined in Eq. (4i. These frequencies, which can be obtained easily with a wkB code, ${ }^{12}$ have been used successfully as initial guesses for the shooting code. In the absence of inhomogeneity, we have found that only damped shear-Alfuen eigenmodes exist in the sheared slab. However, they become less stable when 8 increases. The localization of the mode near the rational surface is due to the Larmor radius effects as well as ion Landau damping.

A 2 1/2-Dimensional $\left(x, y, v_{x}, v_{y}, v_{z}\right)$ finite- $B$ code ${ }^{9}$ has been used for the investigation. The code is based on the Darwin formulation of Maxwell's equations for $w \ll k c$, in which the transverse displacement current in 
Arpere's law is neglected. The compression along the external magnetic field has also been ignored in the code, in accordance with the usual tokamak approximation for a low-B plasma. For the particle pushing, the exact Aynamics for the ions are kept, whereas the electrons are treated as guiding center particles. With the additional assumptions of $\omega \ll$ wpe and $k_{\|} \ll k_{1}$ ' the field equations used in the code are $\nabla_{\phi}^{2}=-4 \pi p$ and $\nabla^{2} A_{\|}=$ $-(4 \pi / G) J J_{\|}$, where $p$ is the charge density and $J_{\|}$is the parellel current. The electric field can be calculated by $E=-\nabla \phi=(1 / c) \partial A / \partial t$ and the magnetic Eield by $B=\nabla \times A_{A}$.

The particle simulation has been carried out in a simple slab geometry with $I_{x} \times I_{y}=64 \Delta \times 32 \Delta$, where $\Delta$ is the grid size. The external magnetic Eield is in the $z$ direction. The potentials, $\phi$ and $A_{\|}$, are assumed to be periodic in $y$ and vanish at the boundaries in $x$. The shear is generated selfconsistenily by a uniform current $w / v_{\text {te }}=0.05$ in the $z$ direction and, therefore, the rational surface is located at the middle of the system in $x$, i. e., $x=0$. The other simulations parameters are: $m_{e} / m_{i}=1 / 400, \tau=4$, ave. no. density $=16 / \mathrm{s}^{2}$, particle size/ $\Delta=1.5, \lambda_{D e} / \Delta=1, \omega_{C i} / \omega_{p t}=0.2$, $c / v_{t e}=4, w_{c i} \Delta t=0.08, B=0.48, L_{s} / \rho_{s}=273, k_{y} \rho_{s}=1.0 \mathrm{~m}$, where $m=0$, $\pm 1, \pm 2, \ldots$, and $\omega^{\star}=0,1, e .$, no spatial inhomogeneity. The simulation has been carried out for $\omega_{c 1} t=0-166$.

In the simulation, we have observed that $k_{y} p_{s}=1.0$ modes are the dominant fluctuations. The measured frequency spectra for the corresponding $\phi$ and $\lambda_{\|}$taken at the posttions where the amplitude is the 
Largest, are shown in Fig. 1, glong with the spatial structures obtained shrough the use of spatial sorrelition function. 13 Also shown in Fig. 1 is the maximum amplitude reached in the simulation for $\phi$ and $A_{\|}$. The time avolution of these modes indicates that what we have observed is not the frowth of an instability. Instead, it is the result of the spatial redistribution of the fielr. energy. The theoretical eigenfrequency for this case, obtained Erom Eqs. (3) and (4). is $w / w_{c i}= \pm .0045-.012 i$. The real part agrees rather well with the measurements. The deviation is due to the resolution of the numerical data as well as the nonlinear effects, which will he fiscussed later. The theoretical mode structures are plotted in Fig. 1. again, there is a good agreement with the simulation results. Therefore, we can sonclude that these fluctuations are indeed shear-alfven eigenmodes and their amplitude is apparently determined by the balance between the damping rate and the Chetenkov emission as well as mode coupling. When a larger system $(64 \Delta \times 64 \Delta)$ is used in the simulation, $k_{y} \rho_{s}=1$ modes are found to remain dominant. This is consistent with the results from the mode equations, which give no localized solutions for $k_{y} \rho_{g}=0.5$ modes. It should be noted here that the existence of a small electron drift has negligible affect on the theoretical resules.

In the sinulations, we have also observed the fomation of magnetic islands as well as the appearance of a $y$ irdependent eddy current, $\Delta J_{0}(x)$. near the rational surface, as shown in Eig. 2. The eddy current, which is nonlinear in nature and is in the direction opposite to the zeroth-order current, acts to nullify the shear near the rational surface. Consequently, magnetic Islands emerge in the shear-free region as the result of finite amplitude osclilations of the eigennodes. These islands, as we know, can casse rapid electron energy transport across the rational surface. 
The existence of the eddy current can be verified theoretically, Using the second-order drift kinetic equation for the electron, we obtain, after some algebra, the quasilinear momentun equation as

$$
s \partial v_{110}^{(2)} / \partial t=\partial A_{10}^{(2)} / \partial t+K^{\prime}\{x\}
$$

whyre $V_{10}^{(2)}$ and $A_{10}^{(2)}$ are the $Y$-averaged secondmorder fluid velocity and vector potential, respectively, $s=\left(c / v_{t e}\right)\left(m_{e} / \pi_{1}\right)^{1 / 2}$

and

$$
\begin{aligned}
K(x) & =2 \sqrt{b} \operatorname{Im}\left\{-A_{n} \phi^{*}+\left(v_{A} / \nabla_{t e}\right)^{2}\left(A_{\|} "-b_{s} A_{\|}\right)\right. \\
& \left.-2\left\{\xi_{e}^{2}-V / Z^{\prime}\left(\xi_{e}\right)\right\}\left(\phi^{\prime}-b_{s} \phi\right) A_{\|}^{*}\right\} .
\end{aligned}
$$

In this equation, length and time are in the units of $\rho_{s}$ and $w_{c i}$ ' respectively, and the linear potentials are normalized by $T_{e} / e$. In arriving at the expression for $k(x)$. Dg. (1) and (2) in the limit of cold ions have been used. Erom Ampere's law, which gives $\delta^{2}\left(A_{10}^{(2)}\right) \cdots=s_{n 0}^{(2)}$ with $j=$ c/upe $\rho$, the piution of Eg. (5) is

$$
s \partial v_{10}^{(2)} / \partial t=R^{\prime}(x)-(1 / 2 \delta) \int_{-\infty}^{\infty} d \zeta \pi^{\prime}(\zeta) \exp (-|x-\zeta| / \delta) .
$$

Substituting the resultg Exom Eqs. (3) and (4) into Bq. (6), we then obtain the second oxder eddy current due to the presence of linear eigermodes, as shown in Fig. 2, where $J_{10}^{(2)}=-\operatorname{env}_{10}^{(2)}$. It compares very well Iith the 


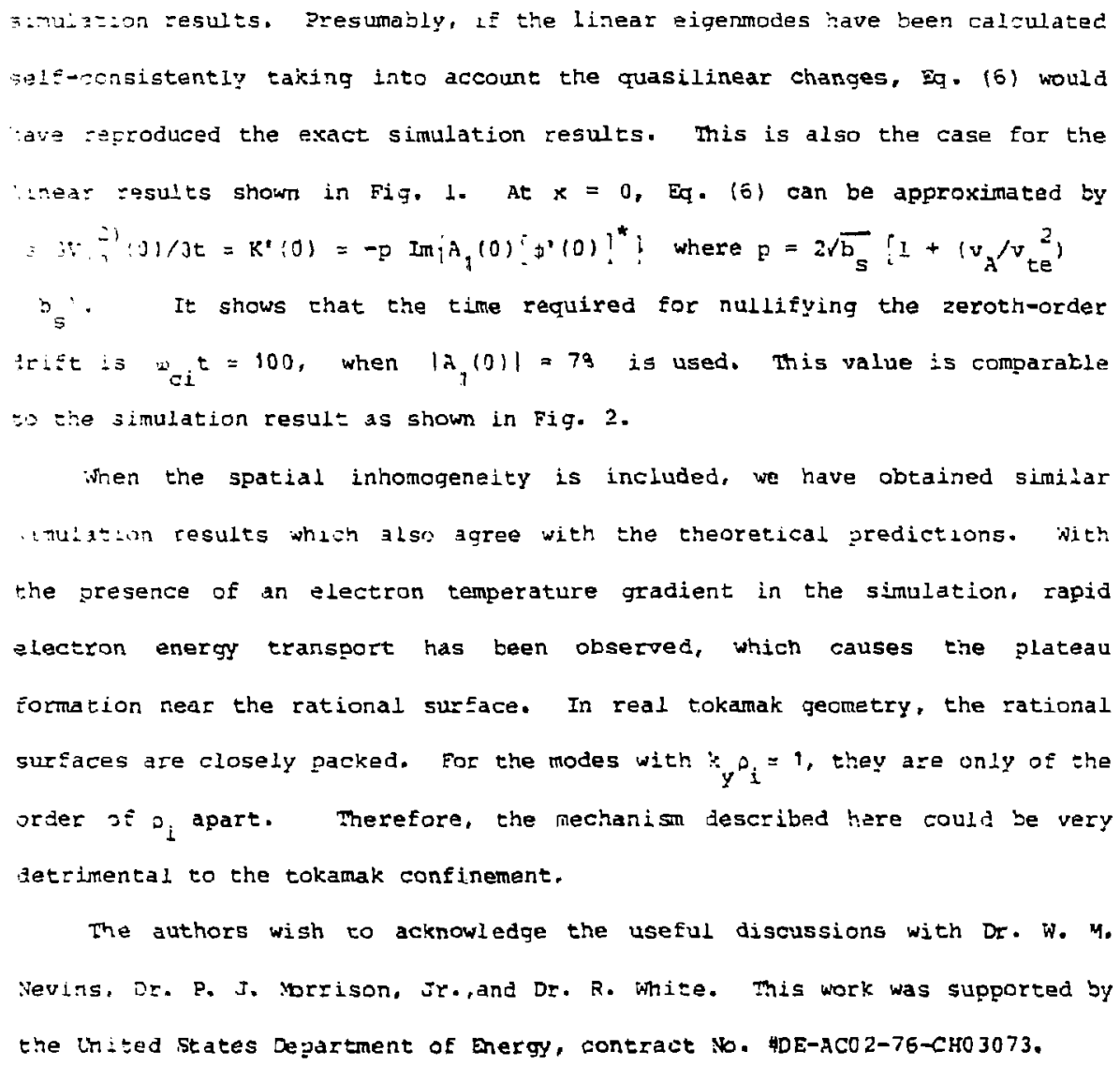


References

1. J. D. Callen, Phys, Rev. Iett. 39 (1977) 1540.

2. x. Wluig, S. D. Iizshman, and J. C. Whitson, Thys. Rev. Iett. 43 (1979) 582 .

3. J. F. Drake, N. T. Gladd, C. S. Liu, and C. L. Chang, Ehys. Rev. Lett. 44 (1980) 994 .

4. K. T. Tsang, J. C. Whitson, J. D. Callen, B. J. Catto, and J. Smith, Phys. Rev. Lett. 41 (1973) 557.

5. Y. C. Lee and L. Chen, Thys. Fev. Lett. 42 (1979) 708.

6. $\quad$ E. Mazucato, Phys. Rev. Lett. 36 (1976) 792 .

7. C. M. Surlo and R. E. Slusher, thys. Rev. Lett. $37(1975) 1747$.

8. 5. J. Zweben, C. R. Wenyuk and R. J. Tayor, Phys. Rev. Lett. 42 (1979) 1270.

9. W. W. Lee and H. Okuda, private communication.

10. S. M. Mahajan, R. D. Hazelelne, H. R. Strauss and D. N. Ross, Phys. Rev. Lett. $41(1978) 1375$.

11. D. J. Morrigon, Jr. ay, W. W. Lee, private communication.

12. R. 9. White, J. Comput, Phys. 31 (1979) 409.

1?. W. W. Lee, W. M. Nevins, H. Okuda, and R. B. White, Phys, Rev. iett. $43(1979) 347$. 

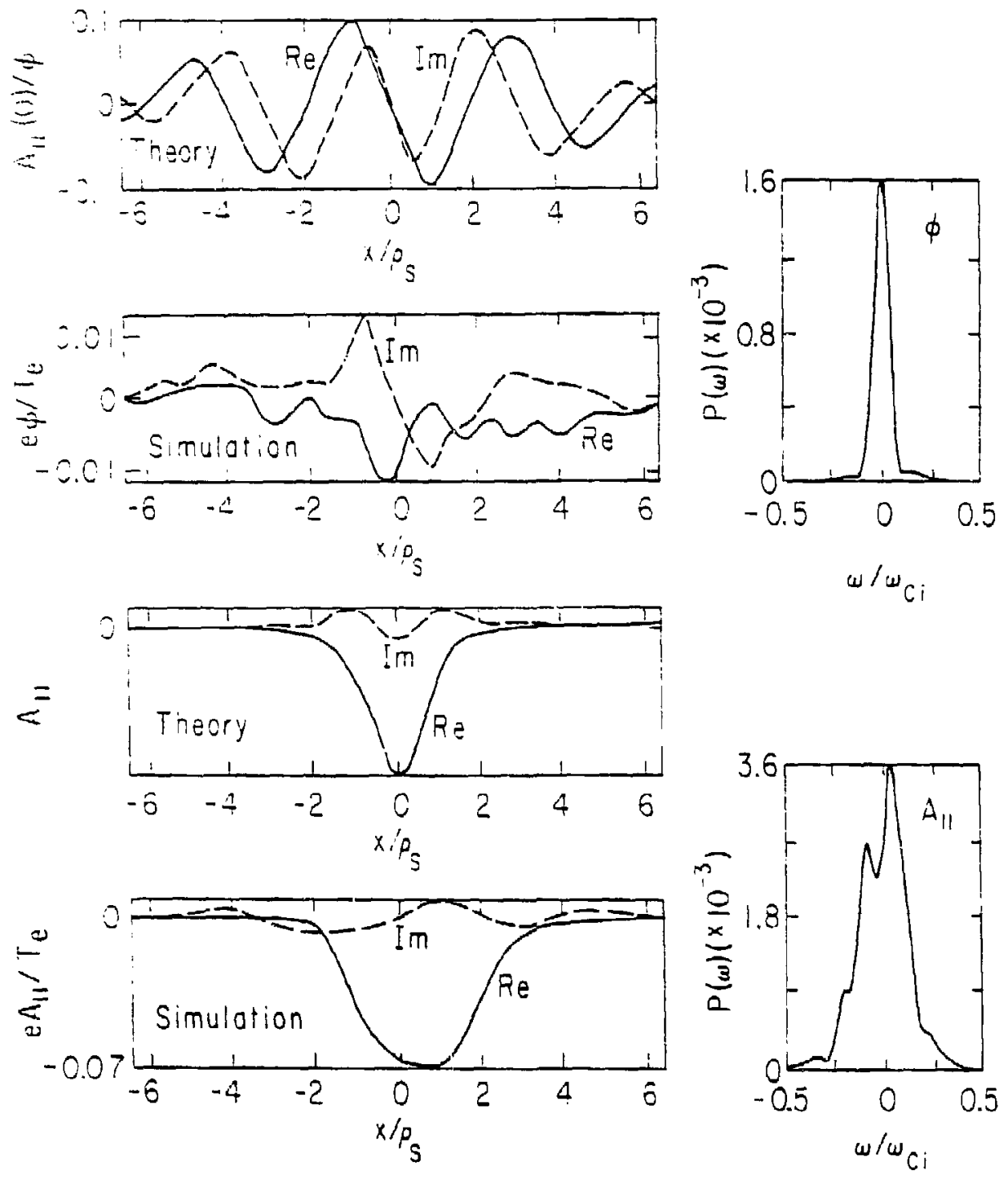

: $2001-502312:$

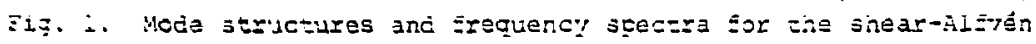
eigermcies. 

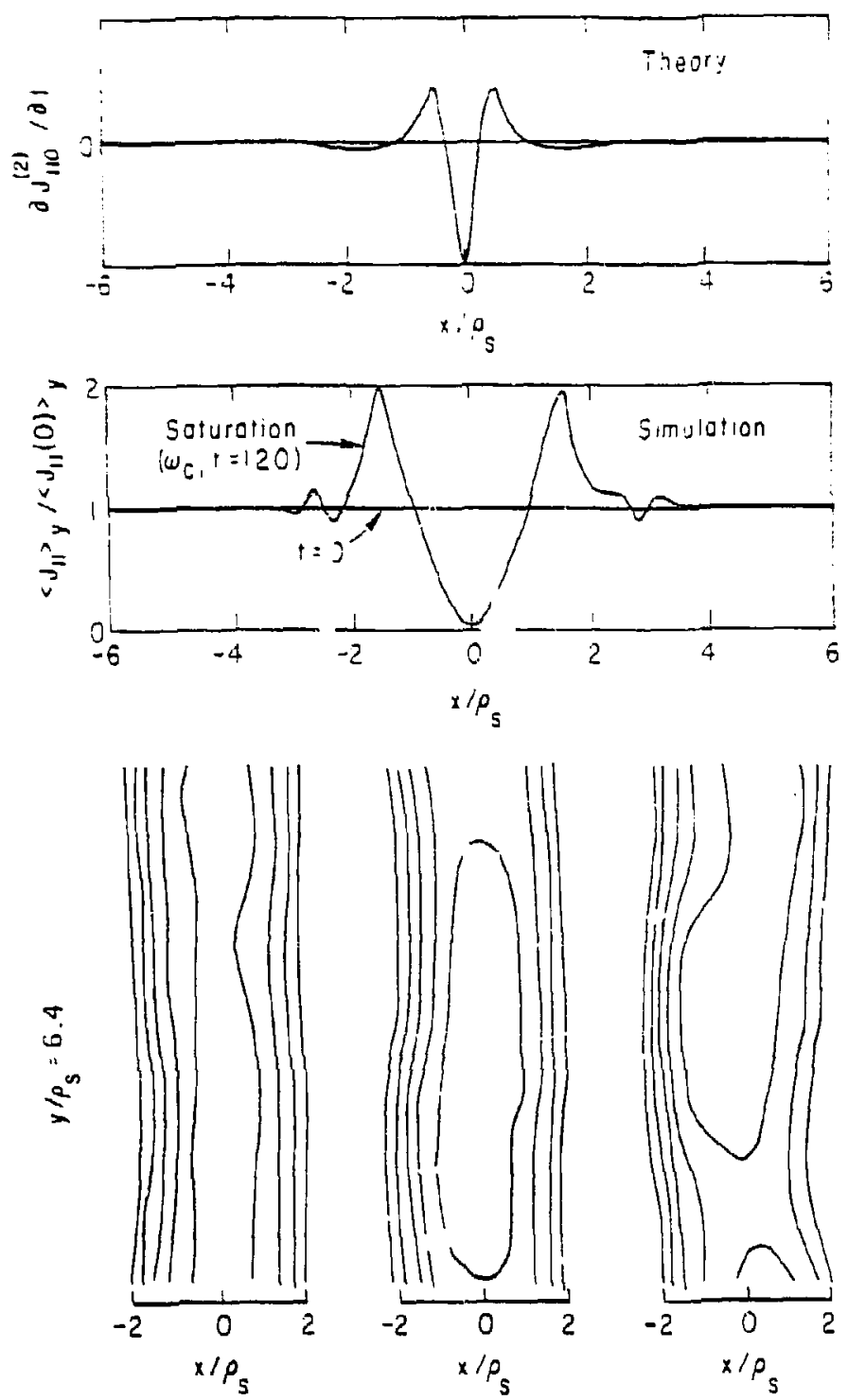

$w_{c_{1}} t=0$

$u_{C i} r=40$

$w_{c i} l=120$

, $20 \mathrm{PT}-30 \geq 2: 1$;

Eig. z. The second-orter scin zurrant and tie =ine

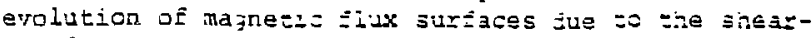
AlEvến elg̣enmodes. 